\title{
Capítulo 2 - Aspectos da natureza da ciência nas pesquisas de Irving Langmuir (1881-1957) relacionadas com a estrutura da matéria
}

\author{
Hélio Elael Bonini Viana
}

\section{SciELO Books / SciELO Livros / SciELO Libros}

VIANA, H.E.B. Aspectos da natureza da ciência nas pesquisas de Irving Langmuir (1881-1957) relacionadas com a estrutura da matéria. In: MOURA, B. A., and FORATO, T. C. M., comps. Histórias das ciências, epistemologia, gênero e arte: ensaios para a formação de professores [online]. São Bernardo do Campo, SP: Editora UFABC, 2017, pp. 37-51. ISBN 978-85-68576-84-7. https://doi.org/10.7476/9788568576847.0003.

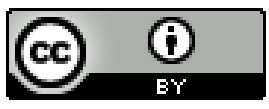

All the contents of this work, except where otherwise noted, is licensed under a Creative Commons Attribution 4.0 $\underline{\text { International license. }}$

Todo o conteúdo deste trabalho, exceto quando houver ressalva, é publicado sob a licença Creative Commons Atribição 4.0.

Todo el contenido de esta obra, excepto donde se indique lo contrario, está bajo licencia de la licencia Creative Commons Reconocimento 4.0. 


\section{ASPECTOS DA NATUREZA DA CIÊNCIA \\ NAS PESQUISAS DE IRVING LANGMUIR \\ (1881-1957) RELACIONADAS COM \\ A ESTRUTURA DA MATÉRIA}

Hélio Elael Bonini Viana

\section{Introdução}

A importância de abordagem histórica da ciência em sala já foi discutida por inúmeros autores - por exemplo, Matthews (1995) e Forato (2009) -, podendo, entre outras benesses, fomentar o pensamento crítico por meio de humanização das ciências e de aulas mais desafiadoras e reflexivas. Todavia, é um equívoco considerar que abordagens focadas na história da ciência resolveriam todos os problemas do ensino de ciências. Aliás, é importante atentar que a veiculação da história da ciência pode resultar em diversos problemas, tais como: erros factuais grosseiros, relações não contempladas entre o processo de produção de conhecimentos na Ciência e o contexto, glorificação do presente e dos seus paradigmas, estímulo da ideia dos conhecimentos científicos como verdades absolutas e entendimento do "progresso" científico como o resultado de descobertas 
realizadas por cientistas geniais. Tal abordagem pode resultar em "[...] uma visão rígida, algorítmica, exata da ciência, reforçado uma interpretação acumulativa, linear do desenvolvimento científico, ignorando crises e revoluções científicas" (BASTOS, 1998, p. 43). Segundo Forato, Martins e Pietrocola (2011), uma das formas para não incorrer nesse tipo de problemas seria a utilização de estudos de casos históricos, os quais poderiam desenvolver a complexa relação entre ciência, tecnologia e sociedade.

De acordo com Porto (2010, p. 174), um estudo de caso histórico pode ser definido como "[...] a análise, com certa profundidade, de algum episódio histórico bem delimitado da História da Ciência". Logo, um estudo de caso pressupõe a abordagem de problemas pertinentes a uma época que influenciaram um cientista - ou um filósofo natural -, ou seja, um relato que viabilize a discussão da natureza sistêmica da natureza científica. Tal abordagem de um estudo de caso está diretamente relacionada com a chamada "nova historiografia da ciência", a qual abarca necessariamente a contextualização das ideias, buscando significados a partir de uma "imersão" em correntes de pensamento de uma determinada época.

Além da sua viabilidade para a elucidação de conceitos científicos, um estudo de caso também pode ser empregado para a discussão de aspectos pertinentes à natureza a ciência (ALLCHIN, 2013; SILVA; MOURA, 2008). Sem perder de vista as discussões acerca de suas vertentes (MOURA, 2014), procurou-se seguir aqui uma abordagem em consonância com a delineada por Douglas Allchin, a qual se destaca, dentre outros pontos, por apresentar estudos de caso históricos como uma atividade de ensino de natureza da ciência em de sala de aula.

Nesse contexto, optou-se pela elaboração de um estudo de caso que contemplasse aspectos da estrutura atômica a 
partir de uma problematização. Dentre as possibilidades encontradas, um estudo embasado em Irving Langmuir (1881-1957) mostrou-se promissor, pelo fato deste cientista ter realizado pesquisas variadas, mostrando interesses tanto pela área básica como pela aplicada. Uma ideia clara da diversidade dos seus interesses pode ser vislumbrada na bibliografia desse cientista americano elaborada por Rosenfeld (1966). De 1906 a 1955, seus trabalhos abordaram assuntos diversos, tais como: reações químicas a altas temperaturas e baixas pressões (1906-1921), efeitos térmicos em gases (1911-1936), estrutura atômica (19191921), emissões termiônicas e superficiais no vácuo (19131937), forças químicas nos sólidos, líquidos e na superfície de filmes (1916-1943), descargas elétricas em gases (1923-1932) e a ciência de ponta (1938-1955). Vale ainda ressaltar que, durante a Primeira Guerra Mundial (1914-1918), Langmuir se envolveu com pesquisas militares, como desenvolvimento de sonares para submarinos e na fixação de nitrogênio em explosivos.

Dessa maneira, tomando por base a maneira de trabaIhar estudos de casos históricos elaborada por Allchin (2013), foram propostas algumas questões reflexivas sobre os trabaIhos de Langmuir relacionados com a estrutura da matéria, com o propósito de orientar um docente para o ensino da natureza da ciência.

\section{As pesquisas de Irving Langmuir sobre estrutura da matéria: um estudo de caso}

Nascido no Brooklin (Nova York), no ano de 1881, Irving Langmuir, o terceiro filho de uma família cujo patriarca era um 
renomado corretor de seguros, teve parte de sua educação básica feita em Paris (1892-1895). Seu irmão, Arthur Langmuir, químico industrial de destaque, foi o seu grande mentor científico em sua infância, despertando-lhe o interesse pela Ciência e por suas aplicações. Como consequência disso, acabou se graduando em Engenharia Metalúrgica pela Universidade de Columbia em 1903. Disposto a prosseguir seus estudos, Langmuir realizou seu doutorado na Universidade de Göttinger (Alemanha), em 1906, sob a orientação de físico-químico Walther Nerst, estudando a dissociação de gases a baixa pressão e alta temperatura.

Voltando aos Estados Unidos, Langmuir começou sua carreira na General Electric Company em 1909, buscando investigar por que as lâmpadas de filamento de tungsténio queimam em um curto espaço de tempo (ROSENFELD, 1966). Enquanto trabalhava para resolver este problema, testando vários gases em um ambiente de baixa pressão - gases inertes como o nitrogênio e argônio foram os que apresentaram os melhores resultados -, outros projetos de investigação emergiram do tratamento desses dados (TAYLOR, 1958). Entre esses projetos está uma proposta para a explicação da estrutura atômica e molecular.

Em setembro de 1916, cerca de três anos antes de iniciar suas publicações sobre a estrutura atômica, Langmuir mostrou entusiasmo por uma apresentação feita por W. H. Bragg, afirmando que "[...] as estruturas dos cristais estudados pelos Braggs nos levam a concepções mais definitivas quanto à natureza da química" (LANGMUIR, 1916, p. 2221). Além dos fenômenos de adsorção e tensão superficial, cujas pesquisas viriam a ter um grande impacto dentro da comunidade científica, o trabalho sobre o uso de raios-x para a elucidação da estrutura cristalina também parece ter fomentado o interesse de Langmuir pelas partículas constituintes de matéria e suas interações 
(LANGMUIR, 1919c). Assim, explorando os estudos sobre o espectro eletromagnético, modelos atômicos e valências - como os de Bohr, Rydberg, Thomson, Rutherford, Kossel e Lewis (LANGMUIR, 1919b), Langmuir conseguiu, de acordo com suas próprias palavras, estender a teoria de Lewis do átomo cúbico - segundo a qual os elétrons de valência, em átomos não combinados, estariam dispostos no vértice de um cubo - propondo representações até o elemento de número atômico 64 (gadolínio-Gd) e empregando-as em uma tentativa de elucidação da estrutura de diferentes partículas, tais como as moléculas de ácidos nitro-oxigenados - Figura 1 (LANGMUIR, 1919a). Para esse empreendimento, Langmuir estabeleceu inicialmente 11 postulados que cobrem o arranjo dos elétrons em átomos isolados e sua partilha preferencial em compostos, tentando deduzir toda a estrutura da química teórica e descritiva. Sobre os seus postulados, Langmuir disse:

Estes postulados marcam o início, creio eu, de uma nova química, uma química dedutiva, na qual podemos propor relações químicas sem cair na intuição química [...]. Eu acho que dentro de poucos anos vamos ser capazes de deduzir 90 por cento de tudo o que está em cada livro sobre química, deduzi-lo como você precisa, a partir de princípios comuns simples, conhecendo fatos definitivos sobre a estrutura do átomo. (1921 apud JENSEN, 1984, p 196)

Para explicar a estabilidade dos átomos, bem como a tendência para estabelecer ligações, Langmuir veio a desenvolver, a partir de seus postulados, uma expressão matemática para a regra do octeto, que ajudou a classificar os compostos em completos (covalentes e não covalentes), incompletos (intermetálicos e sais dos elementos "pesados" - por exemplo, cloreto de crômio III, cloreto de cobre II e óxido de zinco) e casos 
excepcionais (cianeto, gás nitrogênio, boranos, entre outros). De acordo com esta regra, a soma dos elétrons de valência disponíveis ( $\Sigma e$ ) pode ser calculada pela expressão: $\Sigma e=8 \mathrm{~N}-2 \mathrm{~B}$ ( $N$ : número de átomos pesados; B: número de ligações covalentes entre os átomos mais pesados). Logo, amoníaco (NH3) tem um total de oito elétrons disponíveis, porque tem um átomo de nitrogênio $(\mathrm{N}=1)$ e apresenta apenas ligações simples entre o nitrogênio e hidrogénio $(B=0)$. De acordo com a concepção de Langmuir, os elétrons negativos estariam nos arredores de um núcleo de carga positiva, compondo assim a estrutura do átomo (LANGMUIR, 1919a; BURY, 1921). Na eletrosfera, os elétrons estariam contidos em celas de mesmo volume, que pertencem às várias camadas concêntricas de espessura igual em torno do núcleo (com a exceção da primeira camada, cada célula pode conter dois elétrons; o número de celas aumenta de acordo com as camadas, começando com duas na primeira), estando em movimento dentro dessas celas estáticas (LANGMUIR, 1919a).
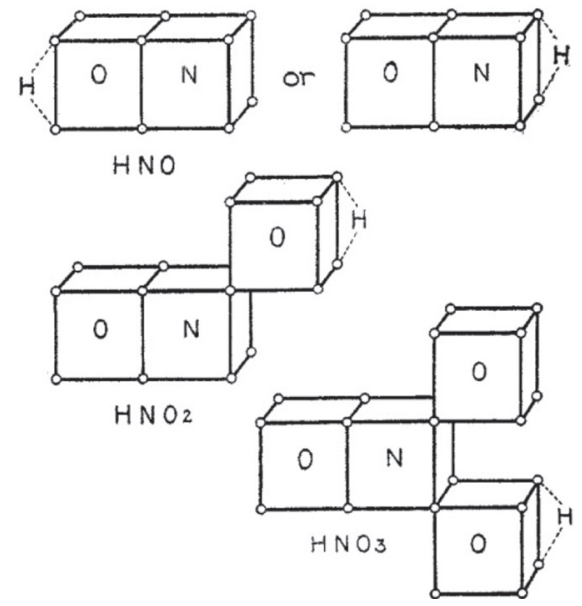

Figura 1 - Modelos de Langmuir empregando átomo cúbico para a representação das moléculas de ácidos nitro-oxigenados

Fonte: Langmuir (1919a, p. 900). 
Em 1921, diante das limitações envolvidas na determinação do número de ligações covalentes com base no número de elétrons de valência disponíveis, Langmuir reduziu seus 11 postulados em apenas três:

1) os elétrons nos átomos tendem a circular o núcleo em sucessivas camadas que contém 2,8,8,18,18 e 32 elétrons respectivamente;

2) dois átomos podem permanecer ligados por uma ou mais duplas [de elétrons] em comum para completar a camada dos dois;

3) a carga residual de cada átomo ou grupo de átomos tendem a um mínimo. (LANGMUIR, 1921 apud JENSEN, 1984, p. 197)

Mesmo tendo alcançado grande sucesso entre recursos acadêmicos e industriais, por conta da fama já alcançada por seus trabalhos, a estrutura atômica proposta com base em postulados sofreu críticas, particularmente de físicos (KOHLER JR., 1974). Sommerfeld se refere a alguns dos postulados de Langmuir como cabalísticos; já Andrade (1927, p. 112) afirma que suas representações continham muito pouco das informações já conhecidas sobre os elétrons, de tal forma que "não é imediatamente claro por que elas são chamadas de elétrons".

Além disso, em 1923, com sua aproximação do modelo dinâmico de Bohr, Lewis posicionou-se contrário à versão que Langmuir desenvolvera a partir dos seus átomos cúbicos:

A prevalência marcante de moléculas em que cada átomo tem sua cota completa de quatro pares de elétrons na camada mais externa levou Langmuir a tentar fazer a regra do octeto absoluto, e até propor uma equação aritmética para determinar, de acordo com esta regra, quando uma dada fórmula representa uma possível substância química. Acredito que o 
entusiasmo por esta ideia está conduzindo-o a um erro, e o que está sendo chamado a nova teoria, a "teoria do octeto", enfatiza demais o que é, afinal, mas uma característica da nova teoria da valência. A regra de oito[elétrons], apesar da grande importância, é menos fundamental do que a regra de dois [elétrons], o que chama a atenção para a tendência de elétrons para formar pares. (LEWIS, 1923 apud JENSEN, 1984, p. 198)

Lewis ainda deixou claro que o trabalho de Langmuir foi desenvolvido de maneira independente:

É um motivo de grande satisfação para mim descobrir que no curso da série de aplicações da nova teoria, conduzido com a maior perspicácia, Dr. Langmuir não foi obrigado a mudar a teoria que eu desenvolvia. Aqui e ali ele foi tentado a considerar certas regras ou tendências como mais universal em seu escopo do que eu considerava-os no meu papel, ou do que eu agora considero-os, mas estas são questões que terão uma oportunidade mais tarde para serem discutidas. A teoria tem sido designada por alguns como a teoria de Lewis-Langmuir, o que implicaria algum tipo de colaboração. Por uma questão de fato, o trabalho de Dr. Langmuir tem sido independente, e tais adições como ele fez com o que foi declarado ou implícito no meu papel deve ser de crédito somente a ele. (KOHLER JR., 1974, p. 60)

Apesar dessas ressalvas, o carisma de Langmuir, evidente em suas palestras e em sua boa relação com os industriais, fez com que sua interpretação sobre as ideias de Lewis - conhecida na época como a teoria Lewis-Langmuir - fosse discutida em vários países entre os anos de 1918 e 1927 (oitenta por cento dos artigos sobre o assunto foram produzidos na Grã-Bretanha, 
Alemanha e Estados Unidos). Contudo, com as rivalidades afloradas após o término da Primeira Guerra Mundial, as tendências nacionalistas tornaram-se acentuadas e resultaram em diferentes respostas para a teoria de Lewis-Langmuir: enquanto nos Estados Unidos as concepções de Lewis expressas em seu livro Valence and the Nature of Chemical Bond (1923) foram largamente divulgadas e discutidas, na Europa as discussões sobre a questão do átomo passaram a focar as contribuições de físicos como Einsten, Schrodinger, Dirac, Pauli e Heisenberg (KOHLER JR., 1975). É importante destacar que, segundo Kohler Jr. (1974), a teoria de Lewis-Langmuir possibilitou os fundamentos para o desenvolvimento da química quântica e da teoria de ressonância.

Em um artigo de 1984, Linus Pauling, ganhador do prêmio Nobel de Química em 1954 e do prêmio Nobel da Paz em 1962, destacou a importância dos trabalhos de Langmuir:

Por outro lado, os dois artigos que Irving Langmuir publicou no Jornal da Sociedade Americana de Química em 1919, num total de 84 páginas, continham várias contribuições e adições significantes à teoria da ligação química por par de elétrons compartilhados. Por alguma razão Lewis foi contra Langmuir, e em minha opinião não deu a ele o devido crédito. (PAULING, 1984, p. 201)

$\mathrm{Na}$ indústria, um sistema periódico com base na estrutura atômica de Langmuir, organizado em função do número de elétrons da camada de valência, foi empregado por Thomas Midgley Jr. para o desenvolvimento de moléculas, tais como tetraetilchumbo e os CFCs, nos Estados Unidos, na primeira metade do século XX. Neste processo, é interessante observar que Midgley, um engenheiro mecânico, na busca por substâncias requeridas pela indústria, fez uso do conceito de grupo para 
investigar possíveis átomos a integrar uma molécula (VIANA; PORTO, 2013).

Após 1921, diante do que Langmuir chamou de "excessiva abstração das discussões sobre a estrutura da matéria" fruto das contribuições oriundas da física quântica -, ele parou de se dedicar às questões do mundo atômico e passou a se dedicar a outros projetos (exemplo: o estudo da adsorção), com aplicações em curto prazo. Em 1950, aposentou-se pela General Eletric Company, passando a atuar como consultor, até o seu óbito (1957).

\section{Debatendo aspectos da natureza da ciência}

Como já foi comentado, a discussão dos aspectos da natureza da ciência no estudo de caso histórico realizado será feita com base em Allchin (2013). Especialista na interface entre história da ciência e ensino, esse autor propõe o ensino da natureza da ciência através de questões reflexivas, as quais auxiliariam a promover respostas reflexivas e articuladas, após o encorajamento do professor, mediante o diálogo entre estudantes com diferentes ideias e perspectivas (ALLCHIN, 2013, p. 177). $\mathrm{Na}$ sequência, estão elencadas algumas questões reflexivas, com os respectivos aspectos da natureza da ciência passíveis de serem ensinados.

Questão reflexiva 1: Quais os fatores que conduziram Langmuir a seus trabalhos sobre a estrutura da matéria?

Conforme relatado, Langmuir construiu sua concepção atômica e molecular partindo de um problema tecnológico evitar que as lâmpadas de tungstênio queimassem facilmente - e, posteriormente, articulando de diversas pesquisas, dentre 
elas as de Lewis e da família Bragg. A maneira como Langmuir estendeu o modelo do átomo cúbico de Lewis é, assim, um artefato da sua imaginação científica através da articulação de pesquisas científicas.

Sendo assim, com esta questão, podem ser discutidos os seguintes aspectos da natureza da ciência: o papel da imaginação científica na representação de partículas; a ciência como construção humana e a influência/colaboração entre cientistas; a abordagem experimental, instrumentos científicos e inovações tecnológicas no desenvolvimento da ciência; o papel da observação na ciência; o raciocínio analógico e o contexto cultural, econômico e tecnológico da ciência.

Questão reflexiva 2: Como explicar o fato de Langmuir ter proposto a regra do octeto?

Também por ter uma formação em engenharia, Langmuir pensava de maneira pragmática, buscando soluções "simples" para problemas tecnológicos e científicos. Dessa maneira, concatenando trabalhos de outros cientistas com a sua própria visão sobre o modus operandi da ciência, Langmuir chegou a uma expressão matemática para a regra do octeto, a qual possibilitaria entender o modo como os átomos se ligam. A partir de críticas, o arcabouço teórico que sustentava essa regra passou por adequações. Desvirtuada de sua expressão matemática e de contexto, a regra do octeto é tratada hoje como um dogma pelos livros didáticos de química, nos diferentes níveis de ensino.

Sendo assim, essa questão permite a discussão de importantes pontos da natureza da ciência como o poder heurístico da regra do octeto, o papel da indução e da dedução na ciência, se a ciência é uma verdade absoluta, mudança conceitual e as perspectivas teóricas na intepretação de dados. 
Questão reflexiva 3: Por que os trabalhos de Langmuir tiveram uma grande aceitação inicial, tanto na academia quanto na indústria?

Por trabalhar na indústria e também ter tido uma sólida formação acadêmica - Langmuir fez o seu doutorado com o renomado físico-químico alemão Walter Nerst -, Langmuir tinha circulação em diferentes meios. Além disso, Langmuir era carismático e um excelente palestrante. Logo, essa questão exemplifica outro aspecto da natureza da ciência, trazendo à tona a importância da comunicação científica dentro da ciência.

Questão reflexiva 4: Por que os méritos de Langmuir relacionados à estrutura dos materiais foram minimizados - para não dizer extirpados - em detrimento de outros cientistas?

Diante da crítica de físicos, Langmuir parou de se dedicar à estrutura da matéria após 1921. Parte dessas críticas eram movidas pelo fato de Langmuir não fazer parte de uma comunidade e por não partilhar de muitos pressupostos de uma área que estava em franca evolução no período, a física quântica. Soma-se a esse fato a influência de posturas nacionalistas - como da Alemanha e da Grã-Bretanha -, as quais foram se mostrando contrárias à teoria de Langmuir com o tempo. Desse modo, essa questão pode discutir as tradições de pesquisa, a influência do nacionalismo na ciência e o progresso não cumulativo da ciência.

Questão reflexiva 5: Por que Langmuir parou de se dedicar a estudos sobre a estrutura da matéria após 1921?

Com o aumento da complexidade - e também da abstração - das discussões sobre a estrutura das partículas, Langmuir passou a se dedicar a projetos capazes de proporcionar respostas em curto prazo, atendendo, assim, às demandas do mercado. Nesse interim, essa questão proporciona a discussão do conhecimento prático versus teórico e também do contexto cultural, econômico e tecnológico da ciência. 


\section{Considerações finais}

Os trabalhos de Langmuir, pela diversidade temática, propiciam um estudo de caso histórico com inúmeras questões pertinentes à natureza da ciência para serem discutidas em sala de aula. Neste trabalho, foram propostas algumas dessas questões, seguindo o procedimento elaborado por Allchin, capazes de promover a habilidade de pensar cientificamente, estimulando a reflexão sobre a natureza da ciência.

Como perspectiva futura, pretende-se utilizar este estudo de caso histórico, juntamente com as questões reflexivas, na formação inicial e continuada de professores. Através da colaboração dos envolvidos, as questões reflexivas poderão ser aperfeiçoadas - ou mesmo serem elaboradas novas perguntas -, para que novos aspectos da natureza da ciência possam ser discutidos.

\section{Referências}

ALLCHIN, Douglas. Teaching the Nature of Science Perspectives e Resources. Saint Paul, MN: SHiPS Education Press, 2013.

ANDRADE, Edward Neville da Costa. The Structure of the Atom. New York: Harcourt, Brace \& Co., 1927.

BASTOS, Fernando. História da ciência e pesquisa em Ensino de Ciências: breves considerações. In: NARDI, Roberto (Org.). Questões atuais no Ensino de Ciências. São Paulo: Escrituras, 1998. p. 43-52.

BURY, Charles R. Langmuir's Theory of The Arrangement of Eletrons in Atoms and Molecules, Journal of the American Chemical Society, n. 43, p. 1602-1609, 1921. 
FORATO, Thaís Cyrino de Mello. A natureza da Ciência como saber escolar: um estudo de caso a partir da história da luz. 2009. v.1 204 p. Tese (Doutorado em Educação) - Faculdade de Educação, Universidade de São Paulo, São Paulo, 2009.

FORATO, Thaís Cyrino de Mello; MARTINS, Roberto de Andrade; PIETROCOLA, Maurício. Historiografia e natureza da Ciência na sala de aula. Caderno Brasileiro de Ensino de Física, v. 28, n. 1, p. 27-59, abr. 2011.

JENSEN, William B. Abegg, Lewis, Langmuir, and the octet rule. Journal of Chemical Education, n. 61, p. 191-200, 1984.

LANGMUIR, Irving. The Constitution and Fundamental Properties of Solids and Liquids. Journal of the American Chemical Society, n. 38, p. 2221-2295, nov. 1916.

. The Arrangement of Electrons in Atoms and Molecules. Journal of the American Chemical Society, v. 41, n. 6, 868-934, jun. 1919a.

. The Structure of Atoms and the Octet Theory of Valence. Proceedings of the National Academy of Science, v. 5, n. 7, p. 252-259, jul. 1919b.

. Isomorphism, Isosterism, and Covalence. Journal of the American Chemical Society, v. 41, n. 10, p. 1543-1559, oct. 1919c.

LEWIS, Gilbert Newton. Valence and the Nature of the Chemical Bond. New York: Chemical Catalog Company, 1923.

KOHLER JR., Robert E. Irving Langmuir and the "Octet" Theory of Valence. Historical Studies in the Physical Sciences, v. 4, p. 39-87, 1974. 
. The Lewis-Langmuir Theory of Valence and the Chemical Community, 1920-1928. Historical Studies in the Physical Sciences, v. 6, p. 431-468, 1975.

MATTHEWS, Michael R. História, Filosofia e ensino de Ciências: a tendência atual de reaproximação. Caderno Catarinense de Ensino de Física, v. 12, n. 3, p. 164-214, 1995.

MOURA, Breno A. O que é a natureza da Ciência e qual a sua relação com a História e a Filosofia da Ciência? Revista Brasileira de História da Ciência, v. 7, n. 1, p. 32-46, 2014.

PAULING, Linus. G. N. Lewis and the chemical bond. Journal of Chemical Education, v. 61, n. 3, p. 201-203, 1984.

PORTO, Paulo A. História e Filosofia da Ciência no Ensino de Química: em busca dos objetivos educacionais da atualidade. In: SANTOS, Wildson Luiz Pereira dos; MALDANER, Otávio Aloísio (Org.). Ensino de Química em Foco. Ijuí: Editora Unijuí, 2010. p. 159-180.

ROSENFELD, Albert. The Quintessence of Irving Langmuir. 1. ed. New York: Pergamon Press, 1966.

SILVA, Cibelle Celestino; MOURA, Breno Arsioli. A natureza da ciência por meio de episódios históricos: o caso da popularização da óptica newtoniana. Revista Brasileira de Ensino de Física, v. 30, n. 1, p. 1602-1610, 2008.

TAYLOR, Henry. Irving Langmuir 1881-1957. London: Biographical Memoirs of Fellows of the Royal Society, 1958.

VIANA, Hélio Elael Bonini; PORTO, Paulo Alves. Thomas Midgley, Jr., and the Development of New Substances: A Case Study for Chemical Educators. Journal of Chemical Education, v. 90, n. 12, p. 1632-1638, 2013. 